\title{
Parásitos Gastrointestinales en Reptiles en Cautiverio en Lima Metropolitana
}

\author{
Gastrointestinal Parasites in Captive Reptiles in Metropolitan Lima \\ Laura Chávez C. ${ }^{1}$, Enrique Serrano-Martínez ${ }^{1,2}$, Manuel Tantaleán V. ${ }^{1}$, \\ Marco Quispe H. ${ }^{1}$, Gina C. Casas V. ${ }^{1}$
}

\section{Resumen}

El presente trabajo tuvo como objetivo identificar parásitos gastrointestinales en reptiles mantenidos en cautiverio en Lima Metropolitana. Se trabajó con reptiles de los parques zonales Huáscar (Villa El Salvador) y Sinchi Roca (Comas), del zoológico del Colegio El Buen Pastor y del serpentario del Instituto Nacional de Salud. Se recolectaron 133 muestras de heces de Chelonoidis denticulata (89), Boa constrictor (11), Iguana iguana (6), Trachemys scripta (3), Bothrops atrox (5), Bothrops barnetti (3), Epicrates cenchria (3), Crotalus durissus terrificus (2) y de otras nueve especies. Las muestras fueron procesadas mediante los métodos de diagnóstico parasitológico directo, de Ritchie, de Faust y frotis fecal con tinción Ziehl Neelsen. Se encontró Nyctotherus sp (72/89), Balantidium sp (56/89) y Sauricola sp (21/89) en C. denticulata; Alaeuris sp (4/6) y Ozolaimus sp (3/6) en I. iguana; ooquistes de Cryptosporidium sp (35/89) en $C$. denticulata; larvas de Rhabdias sp (2/5) en $B$. atrox y huevos del pentastómido Porocephalus sp (1/3) en E. cenchria. Los resultados demuestran que los reptiles son portadores de varias especies de parásitos, incluyendo especies con potencial zoonótico.

Palabras clave: reptiles; parásitos gastrointestinales; Chelonoidis denticulata; Bothrops atrox; Cryptosporidium; Porocephalus

\section{Abstract}

The aim of the study was to identify gastrointestinal parasites in reptiles kept in captivity in Metropolitan Lima. Reptiles in the Huascar Park (Villa El Salvador) and Sinchi Roca Park (Comas), in the zoo of El Buen Pastor School and in the serpentarium of the Instituto Nacional de Salud were sampled. Stoll samples $(n=133)$ were collected from

\footnotetext{
${ }^{1}$ Grupo SALUVET-UPCH, Facultad de Medicina Veterinaria y Zootecnia, Universidad Peruana Cayetano Heredia, Lima, Perú

${ }^{2}$ E-mail: enrique.serrano@upch.pe
}

Recibido: 20 de enero de 2014

Aceptado para publicación: 17 de setiembre de 2014 
Chelonoidis denticulata (89), Boa constrictor (11), Iguana iguana (6), Trachemys scripta (3), Bothrops atrox (5), Bothrops barnetti (3), Epicrates cenchria (3), Crotalus durissus terrificus (2) and other nine species. The samples were processed by parasitological diagnostic methods: direct, flotation with zinc sulfate, Ritchie and Ziehl Neelsen staining. Results shown Nyctotherus sp (72/89), Balantidium sp (56/89), Sauricola sp (21/89) in C. denticulate; Alaeuris sp (4/6) and Ozolaimus sp (3/6) in I. iguana; oocysts of Cryptosporidium sp (35/89) in C. denticulate, Rhabdias sp larvae (2/5) in B. atrox and eggs of Porocephalus sp (1/3) in E. cenchria. The results showed that reptiles are carriers of several species of parasites, including some of zoonotic potential.

Key words: reptiles; gastrointestinal parasites; Chelonoidis denticulate; Bothrops atrox; Cryptosporidium; Porocephalus

\section{INTRODUCCIÓN}

La tortuga «motelo» Chelonoidis denticulata, es objeto de un activo comercio internacional con el fin de suplir la demanda del mercado de mascotas silvestres (Lada y Brestilav, 2000). Estos animales mantienen los parásitos que obtuvieron en su vida libre y que, bajo ciertas condiciones como el estrés, pueden volverse patógenos y ocasionar la muerte del hospedero (Arrojo, 2002). A pesar de ello, es escasa la información sobre el estado sanitario de estos animales, aunque algunos parásitos gastrointestinales han sido reportados como causas frecuentes de mortalidad y morbilidad (Sánchez et al., 2004).

Algunas especies de parásitos en reptiles son parte de la flora intestinal normal, como aquellos de los géneros Balantidium y Nyctotherus, pero su densidad puede incrementarse por el estrés del cautiverio y causar enfermedad (Chinnadurai y DeVoe, 2009; Jacobson, 2007). Por otro lado, estos protozoos tienen un rol importante en la digestión de celulosa (De Bosschere y Roels, 1999).

Nematodos de la familia Atractidae se encuentran en anfibios, reptiles, mamíferos y peces (Ivashkin y Babaeva, 1973). En tortugas se cree que la infección ocurre después de la madurez sexual, por lo que se cuestiona si la transmisión pudiera ocurrir durante la cópula (Petter, 1966). Salizar y Sánchez (2007) registraron, por primera vez para el Perú el nematodo Angusticecum brevispiculum en Chelonoidis denticulata, parásito común en quelonios en cautiverio y de curso usualmente asintomático (Satorhelyi y Sreter, 1993).

En el país se ha registrado el nematodo Alaeuris caudatus en Iguana iguana, el cual convive con nematodos del género Ozolaimus (Tantaleán, 1998). Además, se ha identificado el nematodo Rhabdias spp y el céstodo Ophiotaenia calmetti en Boa constrictor (Sánchez et al., 2004). Los adultos de Rhabdias spp parasitan los pulmones, pudiendo llegar a inducir la producción de mucosidad, dificultad respiratoria y neumonía (Greiner y Mader, 2001). Ophiotaenia sp es un parásito del intestino delgado, que en grandes números alteran la absorción de nutrientes y ocasionan obstrucción intestinal (Tantaleán y Gozalo, 1985).

Otros parásitos importantes son los pentastómidos como Porocephalus sp. Los adultos de Porocephalus crotali son comunes en vipéridos del genero Bothrops, mientras $P$. clavatus se encuentra en boas (Gárate et al., 2007). Los pentastómidos son parásitos casi exclusivos de los reptiles, pero los carnívoros, primates no humanos y humanos pueden ser hospederos accidentales (Drabick, 
Cuadro 1. Relación de especies de reptiles muestreados en tres zoológicos y un serpentario de Lima Metropolitana

\begin{tabular}{|c|c|c|}
\hline Ubicación & Especie & Población \\
\hline \multirow[t]{4}{*}{ Parque Zonal Sinchi Roca } & Chelonoidis denticulata & 40 \\
\hline & Trachemys scripta & 3 \\
\hline & Iguana iguana & 2 \\
\hline & Boa constrictor & 1 \\
\hline \multirow[t]{2}{*}{ Parque Zonal Huáscar } & Chelonoidis denticulata & 49 \\
\hline & Iguana iguana & 3 \\
\hline \multirow[t]{2}{*}{ Zoológico "El Buen Pastor" } & Iguana iguana & 1 \\
\hline & Boa constrictor & 1 \\
\hline \multirow{14}{*}{$\begin{array}{l}\text { Serpentario del Instituto Nacional } \\
\text { de Salud }\end{array}$} & Bothrops atrox & 5 \\
\hline & Bothrops barnetti & 3 \\
\hline & Bothrops roedingeri & 1 \\
\hline & Bothrops pictus & 1 \\
\hline & Crotalus durissus terrificus & 2 \\
\hline & Bothriopsis chloromelas & 1 \\
\hline & Boa constrictor & 9 \\
\hline & Epicrates cenchria & 3 \\
\hline & Clelia clelia & 2 \\
\hline & Phylodrias sp. & 2 \\
\hline & Eunectes murinus & 1 \\
\hline & Oxyrhopus fitzgeri & 1 \\
\hline & Pseutes sp. & 1 \\
\hline & Spilotes pullatus & 1 \\
\hline
\end{tabular}

1987; Hendrix y Blagburn, 1988). Por otro lado, los reptiles parecen tener especies de Cryptosporidium que son patógenas para una clase específica de animales, pero que pueden tener un curso zoonótico, especialmente en personas con inmunosupresión (Montali, 1999).

El presente trabajo tuvo como objetivo identificar parásitos gastrointestinales en reptiles mantenidos en cautiverio en centros de crianza de la zona de Lima. Esto permitirá establecer medidas sanitarias que eviten infecciones en animales en cautiverio y el posible impacto negativo en la salud pública.

\section{Materiales y Métodos}

\section{Lugar de Estudio}

Se trabajó con el total de la población de reptiles mantenidos en cautiverio en los parques zonales Sinchi Roca y Huáscar, en 
Cuadro 2. Frecuencia de parásitos gastrointestinales en reptiles mantenidos en cautiverio en Lima Metropolitana

\begin{tabular}{|c|c|c|c|}
\hline & \multirow[b]{2}{*}{ Especie } & \multicolumn{2}{|c|}{ Reptiles } \\
\hline & & $\begin{array}{l}\text { Total } \\
\text { (n) }\end{array}$ & $\begin{array}{c}\text { Positivos } \\
(\%)\end{array}$ \\
\hline \multirow[t]{3}{*}{ Tortugas } & & 92 & 93.5 \\
\hline & Chelonoidis denticulata & 89 & 95.5 \\
\hline & Trachemys scripta & 3 & 33.3 \\
\hline \multirow[t]{2}{*}{ Iguanas } & & 6 & 100 \\
\hline & Iguana iguana & 6 & 100 \\
\hline \multirow[t]{6}{*}{ Serpientes } & & 35 & 28.6 \\
\hline & Bothrops atrox & 5 & 40.0 \\
\hline & Bothrops barnetti & 3 & 33.3 \\
\hline & Crotalus durissus terrificus & 2 & 50.0 \\
\hline & Boa constrictor & 11 & 27.3 \\
\hline & Epicrates cenchria & 3 & 33.3 \\
\hline Total & & 133 & 76.3 \\
\hline
\end{tabular}

los distritos de Comas y Villa El Salvador, respectivamente; así como del zoológico del Colegio El Buen Pastor en Los Olivos y del Serpentario del Instituto Nacional de Salud ubicado en el distrito de Chorrillos, de la ciudad de Lima. La distribución de la población de reptiles se muestra en el Cuadro 1.

Se registró la especie, sexo, edad y procedencia (cautiverio o vida libre) de cada individuo. Muestras de heces fueron recolectadas directamente de los ambientes de los animales. Las muestras obtenidas $(n=133)$ fueron llevadas al Laboratorio de Parasitología de la Facultad de Medicina Veterinaria y Zootecnia de la Universidad Peruana Cayetano Heredia, Lima.

\section{Métodos Coproparasitológicos}

Se utilizaron cuatro métodos coproparasitológicos: directo, de Faust, de Ritchie modificado y frotis fecal con tinción Ziehl-
Neelsen (Henricksen y Pohlenz, 1981). Los huevos y larvas de cada especie fueron identificadas según sus características morfológicas y métricas (Tantaleán et al., 1983; Tantaleán y Gozalo, 1985; Tantaleán, 1998; Sánchez et al., 2004). Se consideró muestra positiva si presentaba uno o más parásitos y negativa si no presentaba parásitos. Los ooquistes de Cryptosporidium se reconocieron por el color que toman con la fucsina, así como por su forma y tamaño (Henricksen y Pohlenz, 1981).

\section{Análisis de Datos}

Los datos obtenidos fueron resumidos mediante estadística descriptiva para determinar la frecuencia de individuos positivos y la frecuencia de parásitos identificados. La asociación entre las variables independientes sexo, edad y procedencia y la presentación de infecciones parasitarias fue determinada mediante la prueba de Chi Cuadrado. 
Cuadro 3. Parásitos gastrointestinales en reptiles mantenidos en cautiverio en Lima Metropolitana

\begin{tabular}{|c|c|c|c|c|}
\hline \multirow{2}{*}{ Grupo } & \multirow{2}{*}{ Especies } & \multirow{2}{*}{ Parásitos } & \multicolumn{2}{|c|}{ Positivos } \\
\hline & & & (n) & $(\%)$ \\
\hline \multirow{8}{*}{$\begin{array}{l}\text { Tortugas } \\
(n=92)\end{array}$} & \multirow[t]{7}{*}{ Chelonoidis denticulada (89) } & Nyctotherus sp & 72 & 80.9 \\
\hline & & Balantidium sp & 56 & 62.9 \\
\hline & & $\begin{array}{l}\text { Huevos tipo } \\
\text { Strongylus sp }\end{array}$ & 28 & 31.5 \\
\hline & & Sauricola sp & 21 & 23.6 \\
\hline & & Atractidae sp & 18 & 20.2 \\
\hline & & Angusticaecum sp & 10 & 11.2 \\
\hline & & Cryptosporidium $\mathrm{sp}$ & 35 & 39.3 \\
\hline & Trachemys scripta (3) & Nyctotherus sp & 1 & 33.3 \\
\hline \multirow{2}{*}{$\begin{array}{l}\text { Iguanas } \\
(\mathrm{n}=6)\end{array}$} & \multirow[t]{2}{*}{ Iguana iguana 6) } & Alaeuris sp & 4 & 66.7 \\
\hline & & Ozolaimus sp & 3 & 50.0 \\
\hline \multirow{16}{*}{$\begin{array}{l}\text { Serpientes } \\
(\mathrm{n}=35)\end{array}$} & \multirow[t]{2}{*}{ Bothrops atrox (5) } & Rhabdias sp & 2 & 40.0 \\
\hline & & Ophiotaenia $\mathrm{sp}$ & 1 & 20.0 \\
\hline & Bothrops barnetti (3) & Ophiotaenia sp & 1 & 33.3 \\
\hline & Bothrops roedingeri (1) & Cryptosporidium $\mathrm{sp}$ & 1 & 33.3 \\
\hline & Bothrops pictus (1) & Cryptosporidium $\mathrm{sp}$ & 1 & 33.3 \\
\hline & Crotalus durissus (2) & Ophiotaenia $\mathrm{sp}$ & 1 & 50.0 \\
\hline & \multirow[t]{2}{*}{ Boa constrictor $(11)$} & Ophiotaenia $\mathrm{sp}$ & 1 & 9.1 \\
\hline & & Cryptosporidium sp & 2 & 18.2 \\
\hline & Epicrates cenchria (3) & Porocephalus sp & 1 & 33.3 \\
\hline & Clelia clelia (2) & - & 0 & 0 \\
\hline & Phylodrias $s p(2)$ & - & 0 & 0 \\
\hline & Bothriopsis chloromelas (1) & - & 0 & 0 \\
\hline & Eunectes murinus (1) & - & 0 & 0 \\
\hline & Oxyrhopus fitzgeri (1) & - & 0 & 0 \\
\hline & Pseutes sp (1) & - & 0 & 0 \\
\hline & Spilotes pullatus (1) & - & 0 & 0 \\
\hline
\end{tabular}

\section{Resultados y Discusión}

Se encontraron parásitos gastrointestinales en el $76.3 \%$ de la población de reptiles en los cuatro establecimientos en estudio
(Cuadro 2). Se identificaron 12 especies de parásitos, correspondiendo a los protozoos Nyctotherus sp, Balantidium sp y Cryptosporidium $\mathrm{sp}$, los nematodos Sauricola sp, Angusticaecum sp, larvas de la familia Atractidae, huevos tipo Strongylus, 
Alaeuris sp, Rhabdias sp y Ozolaimus sp, el cestodo Ophiotaenia sp y el pentastómido Porocephalus sp (Cuadro 3).

No se encontraron asociaciones significativas en el análisis de las variables sexo, edad y procedencia, posiblemente debido al bajo número de muestras en algunos de los grupos.

Dentro de las 17 especies de reptiles en estudio, Chelonoidis denticulata e Iguana iguana destacan por ser las especies con mayor frecuencia de parásitos $(95.5 \%$ [85/ 89] y $100 \%$ [6/6], respectivamente). En el caso de las serpientes, de las 15 especies en estudio, solo se encontraron parásitos en 5 de ellas, pero debe tenerse en cuenta el pequeño número de serpientes por especie y que la mayoría procedía del serpentario del Instituto de Salud, donde reciben la debida atención sanitaria.

Se observó una alta presencia de Nyctotherus spp (80.9\%) y Balantidium spp (62.9\%) en C. denticulata. Estos protozoos ciliados forman parte de la flora normal del tracto digestivo de los quelonios y tienen un papel importante en la digestión de la celulosa (De Bosschere y Roels, 1999). Se conoce que la densidad de estos parásitos puede incrementarse por el estrés asociado con el cautiverio o enfermedades concurrentes (Chinnadurai y Devoe, 2009). Estos parásitos se encontraron mayormente en la forma quística, aunque también como trofozoítos.

El $23.6 \%$ de $C$. denticulata dió positivo al nematodo Sauricola sp. Tantaleán et al. (1983) reportaron S. sauricola en el intestino delgado en tortugas $C$. denticulata de Loreto, Perú, en tanto que Bursey y Brooks (2011) reportaron Rhinoclemmys annulata $y$ R. pulcherrima en el Área de Conservación de Guanacaste, Costa Rica. Por otro lado, el 20.2 y $11.2 \%$ de las muestras de $C$. denticulata fueron positivas para larvas de Atractidae spp y Angusticaecum spp, respectivamente. Salizar y Sánchez (2007) re- portaron A. brevispiculum en tortugas $C$. denticulata provenientes de Ucayali y el Parque Genaro en Loreto, Perú, en tanto que Tantaleán et al. (1983) registraron en estas tortugas una especie desconocida de Angusticaecum sp para el Perú.

En las muestras de Iguana iguana se encontraron huevos de nematodos oxiuridos de los géneros Alaeuris y Ozolaimus, familia Pharyngodonidae, con frecuencias de 66.7 y $50 \%$, respectivamente. $\mathrm{Al}$ respecto, la especie de A. caudatus ha sido reportada por Tantaleán (1998) en I. iguana en Tumbes, Perú, mientras que Dyer et al. (1999) registraron A. vogelsangi en el intestino grueso de un ejemplar adulto de I. iguana en Costa Rica. Por otro lado, especies de $O$. megatyphlon han sido reportadas por Tantaleán (1976) en iguanas de Tumbes, mientras que Arrojo (2002) reportó $O$. megatyphlon y $O$. cirratus en el ciego intestinal de iguanas decomisadas en un mercado de Lima, Perú.

Larvas de Rhabdias spp se hallaron en 40\% de muestras de Bothrops atrox; hallazgo que ratifica el reporte de Tantaleán y Gozalo (1985) en B. atrox capturados en Loreto, Perú. Por otro lado, Sánchez et al. (2004) registraron Rhabdias spp en pulmones de Boa constrictor, Epicrates cenchria y Corallus caninus. En el presente estudio, ninguno de los ejemplares $B$. atrox positivos para Rhabdias presentaba signos clínicos y las larvas se encontraron en muestras de heces, pues no se evaluó su presencia a nivel pulmonar.

Huevos de Ophiotaenia spp se encontraron en $1 / 5,1 / 3,1 / 2$ y $1 / 11$ muestras de $B$. atrox, B. barnetti, Crotalus durissus terrificus y $B$. constrictor, respectivamente. En el Perú, Tantaleán y Gozalo (1985) reportaron por primera vez Ophiotaenia sp en el intestino delgado de $B$. atrox, mientras que en 1992 identificaron la especie $O$. calmetti. 
Por otro lado, Sánchez et al. (2004) reportaron una especie no identificada de Ophiotaenia en B. constrictor, E. cenchria y $C$. caninus, sugiriendo que podría ser causa de morbilidad y mortalidad en animales cautivos jóvenes y adultos.

Únicamente se encontraron huevos del pentastómido Porocephalus en Epicrates cenchria. $P$. crotali se reportó por primera vez en el país en pulmones de Bothrops atrox (Tantaleán y Gozalo, 1985) y, posteriormente, Gárate et al. (2007) identificaron a $P$. stilessi en la serpiente Lachesis muta. Otro estudio realizado en Brasil reportó una especie desconocida de Porocephalus sp en Boa constrictor y $P$. stilessi en Lachesis muta (Oliveira et al., 2008). El género Porocephalus puede causar lesiones en los pulmones y obstrucción de la tráquea, siendo uno de los parásitos más patógenos en serpientes y de importancia en salud pública, debido a que sus larvas pueden encontrarse en las vísceras de humanos.

Cryptosporidium spp se identificaron en $C$. denticulata, Bothrops roedingeri, Bothrops pictus y Boa constrictor. Cryptosporidium spp fue reportado en 14 serpientes con gastritis hipertrófica por Brownstein et al. (1977). Asimismo, Levine (1980) nombraría Cryptosporidium serpentis a los ooquistes que infectaban la mucosa gástrica de reptiles; y más adelante, Hassl y Benyr (2003) identificaron C. parvum y $C$. serpentis en heces de anfibios y reptiles. La presencia de $C$. parvum sugiere que la transmisión zoonótica es posible o que los humanos podrían ser la fuente de infección en reptiles.

El riesgo de zoonosis transmitidas por reptiles es cada vez más preocupante debido al aumento del comercio ilegal de fauna silvestre y la popularidad de los reptiles como mascotas; sin embargo, el potencial zoonótico no está claramente establecido (Montali, 1999).

\section{ConClusiones}

- Se identificaron los parásitos Nyctotherus sp, Balantidium sp, Sauricola sp, larvas de la familia Atractidae, Angusticaecum sp, Alaeuris sp, Ozolaimus sp, Rhabdias sp, Ophiotaenia sp, Porocephalus sp y Cryptosporidium sp.

- Este estudio representa el primer reporte de Nyctotherus sp, Balantidium sp y Cryptosporidium sp en reptiles en el Perú.

- Las frecuencias de parásitos en Chelonoidis denticulata no fueron estadísticamente significativas para el factor sexo, edad y procedencia.

- Los reptiles poseen parásitos gastrointestinales que podrían representar un riesgo para la salud pública.

\section{Literatura Citada}

1. Arrojo L. 2002. Parásitos de animales silvestres en cautiverio en Lima, Perú. Rev Peru Biol 9: 118-120.

2. Brownstein DG, Strandberg JD, Montall RJ, Bush M, Fortner J. 1977. Cryptosporidium in snakes with hypertrophic gastritis. Vet Pathol 14: 606617.

3. Bursey CR, Brooks DR. 2011. Nematode parasites of five species of turtles from the Area de Conservación de Guanacaste, Costa Rica, with description of a new species of Falcaustra. Comp Parasitol 78: 107-119. doi: 10.1654/4431.1.

4. Chinnadurai S, DeVoe R. 2009. Selected infectious diseases of reptiles. Vet Clin Exot Anim 12: 583-596. doi: 10.1016/j.cvex.2009.06.008.

5. De Bosschere H, Roels S. 1999. Balantidium sp and Nyctotherus sp: two common members of the digestive tract flora in Mediterranean tortoises. 
[Internet]. Available in: http:// www.tortoisetrust.org/articles/balantidium.htm

6. Drabick JJ. 1987. Pentastomiasis. Rev Inf Dis 9: 1087-1090.

7. Dyer W, Bunkley-Williams L, Williams E. 1999. Two new Caribbean records of parasitic nematodes collected from reptiles in Puerto Rico: Aplectana pusilla in Amphisbaena bakeri and Alaeuris vogelsangi in Iguana iguana. Caribbean J Sci 35: 158-159.

8. Gárate I, Naupay A, Suyo B, Colquichagua $H$, Rodríguez $E$, Yarlequé A. 2007. Identificación de Porocephalus stilessi (Pentastomida) en la serpiente peruana Lachesis muta. Rev Inv Vet Peru 18: 89-93.

9. Greiner EC, Mader DR. 2001. Parasitology. In: Mader DR (ed). Reptile medicine and surgery. St Louis: Saunders. p 343-364.

10. Hassl A, Benyr G. 2003. Hygienic evaluation of terraria inhabited by amphibians and reptiles: Cryptosporidia, free-living Amebas, Salmonella. Wien Klin Wochenschr 115 (Suppl 3): 68-71.

11. Hendrix CM, Blagburn BL. 1988. Reptilian Pentastomiasis: a possible emerging zoonoses. Comp Cont Educ Pract Vet 10: 46-50.

12. Henricksen SA, Pohlenz JFL. 1981. Staining of Cryptosporidium by a Modified Ziehl-Neelsen technique. Acta Vet Scand 22: 594-596.

13. Ivashkin MV, Babaeva MB. 1973. The internal cycle of viviparus nematodes from the gastrointestinal tract of animals. In: Gagarin VG (ed). Problemy Obshchei i Prikladnoi Gelmintologii. Moscow: Izdatel'stvo. p 61-68.

14. Jacobson ER. 2007. Parasites and parasitic diseases of reptiles. In: Jacobson ER (ed). Infectious diseases and pathology of reptiles. Boca Raton: CRC Press. p 572-597.

15. Lada DM, Bretislav JS. 2000. Description of Eimeria motelo sp. n. (Apicomplexa: Eimeriidae) from the yellow footed tortoises, Geochelone denticulata (Chelonia: Testudinidae), and replacement of Eimeria carinii Lainson, Costa \& Shaw, 1990 by Eimeria lainsoni nom. nov. Mem Inst Oswaldo Cruz 95: 829-832.

16. Levine ND. 1980. Some corrections of coccidian (Apicomplexa: Protozoa) nomenclature. J Parasitol 66: 830-834.

17. Montali RJ. 1999. Important aspects of zoonotic diseases in zoo and wildlife species. Verh Ber Erkrg Zootiere 39: 149-155.

18. Oliveira W, Silva F, Camargo M, Vieira S. 2008. Porocephalus species (Pentastomida) infecting Boa constrictor (Boidae) and Lachesis muta in northeastern Brazil. Biotemas 21: 165-168. doi: 10.5007/ 2175-7925. 2008v21n2p165.

19. Petter AJ. 1966. Equilibre des especes dans les populations de nematodes parasites du colon des tortues terrestres. Mem Museum Nat d'Histoire Naturelle 39: 1-152.

20. Salizar P, Sánchez L. 2007. Nuevos registros de nematodos en dos especies de tortugas (Reptilia: Testudines) en el Perú. Neotrop Helminthol 1: 43-45.

21. Sánchez N, Tantaleán M, Richards $R$, Gálvez H. 2004. Parásitos helmintos en Boa constrictor, Epicrates cenchria y Corallus caninus (Ophidia: Boidae) criadas en cautiverio. Rev Inv Vet Peru 15:166-169.

22. Satorhelyi T, Sreter T. 1993. Studies on internal parasites of tortoises. Parasit Hung 26: 51-55.

23. Tantaleán M. 1976. Contribución al conocimiento de los helmintos de vertebrados del Perú. Biota 10: 435-443.

24. Tantaleán M. 1998. Nuevos registros de nematodes parásitos de animales de vida silvestre en el Perú. Rev Peru Biol 5: 103-104.

25. Tantaleán M, Gozalo A. 1985. Parásitos de Bothrops atrox (Viperidae) de la Amazonía peruana. AMVEAP 20: 11-12.

26. Tantaleán M, Juárez D, Cruz C. 1983. Helmintos nuevos para el Perú. Boletín IMT/UNMSM 3: 1-3. 